\title{
On Physical Layer Security of Double Rayleigh Fading Channels for Vehicular Communications
}

\author{
Yun Ai, Member, IEEE, Michael Cheffena, Aashish Mathur, Member, IEEE, and Hongjiang Lei, Member, IEEE
}

\begin{abstract}
In this letter, we study the physical layer secrecy performance of the classic Wyner's wiretap model over double Rayleigh fading channels for vehicular communications links. We derive novel and closed-form expressions for the average secrecy capacity (ASC) taking into account the effects of fading, path loss and eavesdropper location uncertainty. The asymptotic analysis for ASC is also conducted. The derived expressions can be used for secrecy capacity analysis of a number of scenarios including vehicular-to-vehicular (V2V) communications. The obtained results reveal the importance of taking the eavesdropper location uncertainty into consideration while designing V2V communication systems.
\end{abstract}

Index Terms-Physical layer security, average secrecy capacity, double Rayleigh fading, vehicle-to-vehicle (V2V) communication.

\section{INTRODUCTION}

$\mathbf{P}$ HYSICAL LAYER SECURITY (PLS) has widely been considered to be a potential paradigm to enhance communication secrecy against eavesdropping in wireless communication networks [1]. There is an increasing number of research exploring the physical layer secrecy performance of communication systems over different fading conditions. The average secrecy capacity (ASC) over $\kappa-\mu$ and $\alpha-\mu$ fading channels have been derived in [2] and [3], respectively. The secrecy outage probability (SOP) performance over correlated composite Nakagami- $m$ /Gamma fading channels was investigated in [4]. In [5], the closed-form expressions for SOP were derived by taking into consideration both the impact of fading as well as the eavesdropper's location uncertainty. The effect of protected zone around the source node on ASC in a random wireless network was investigated in [6], which was shown to lead to significant improvement in security.

Vehicular communications enable the information transmission between vehicles or with other objects. Vehicle-to-vehicle $(\mathrm{V} 2 \mathrm{~V})$ communications is playing a key role in increasing road safety and efficiency, which is usually developed as part of the intelligent transportation system (ITS) and lays the foundation for future autonomous transport systems [7]. Meanwhile, lack of secure communications between vehicles might result in abuses or attacks, which can jeopardize ITS efficiency and threaten driving safety [8]. This motivates the research on vehicular communications from the perspective of communication security.

Due to the high mobility of vehicles, the well-known channel models such as Rayleigh, Rician or Nakagami-m,

Manuscript received February 8, 2018; revised April 24, 2018; accepted June 29, 2018. Date of publication MONTH DAY, 2018; date of current version MONTH DAY, 2018. The associate editor coordinating the review of this paper and approving it for publication was S. Ma.

Y. Ai and M. Cheffena are with the Norwegian University of Science and Technology, Norway (e-mail: yun.ai@ntnu.no; michael.cheffena@ntnu.no).

A. Mathur is with the Indian Institute of Technology Jodhpur, India (e-mail: aashishmathur@iitj.ac.in).

H. Lei is with the King Abdullah University of Science and Technology, Saudi Arabia, and also with Chongqing University of Posts and Telecommunications, China (e-mail: leihj@cqupt.edu.cn).

Digital Object Identifier which were initially proposed for stationary communication links, do not fit well for a lot of V2V channel measurements [9]-[11]. Instead, double Rayleigh fading has been proposed as a more precise characterization of the dynamic non line-ofsight (NLOS) vehicular links based on both field measurement and theoretical analysis in [9]-[11] and the references therein, where the double-bounce scattering components caused by scatterers around both the transmitter's and receiver's local environments lead to a cascaded Rayleigh fading processes [11]. Additionally, it has been shown that double Rayleigh distribution also fits various measurements in different suburban outdoor-to-indoor mobile-to-mobile (M2M) communication scenarios as well as for keyhole channels [12].

Motivated by the latest advances in physical layer security analysis [2]-[6] and the importance of security in vehicular communications, we study the secrecy performance of vehicular communications in this letter. Additionally, due to the high mobility of vehicles, and more importantly, the eavesdropper's intention to hide its position, we also take into account of the uncertainty of the eavesdropper's location in our analysis. We represent the location uncertainty with a parameter pair, which enables us to gain more insights on the impact of location uncertainty on the secrecy performance compared to previous results. To the best of the authors' knowledge, the secrecy performance of double Rayleigh fading channel has not been studied from the physical layer perspective, nor can it be approximated by those of other investigated fading distributions [2]-[6]. More specifically, we investigate the secrecy performance of the Wyner's model in the scenario of $\mathrm{V} 2 \mathrm{~V}$ communications under eavesdropper location uncertainty.

Notations: $K_{v}(\cdot)$ denotes the modified Bessel function of second kind with order $v$ [13, Eq. 8.407], $C=-\psi(1)$ is EulerMascheroni constant with $\psi(\cdot)$ being Euler psi function [13, Eq. 8.367], and $G_{p, q}^{m, n}\left(x \mid \begin{array}{l}a_{1}, \ldots, a_{p} \\ b_{1}, \ldots, b_{q}\end{array}\right)$ is the Meijer G-function [13, Eq. 9.343].

\section{Channel And System Models}

We consider the classical Wyner's wiretap model in our analysis [3]. Under Wyner's model, the vehicle $S$ sends confidential information to the desired receiver $D$ (another moving vehicle in $\mathrm{V} 2 \mathrm{~V}$ scenario) whereas the eavesdropper vehicle $E$ tries to intercept the information by decoding its received signal. It assumed that the communication links for V2V communications experience independent double Rayleigh fading and the distance for the legitimate communication (between nodes $S$ and $D$ ) is known to $S$. We also consider the realistic scenario of eavesdropping that the exact distance for the eavesdropper link (between nodes $S$ and $E$ ) is unknown to $S$ due to the vehicle mobility and node $E$ 's intention to hide its exact position; but $S$ has the information that the position of node $E$ is located between ranges $R_{1}$ and $R_{2}$ with equal probability. In $\mathrm{V} 2 \mathrm{~V}$ scenario, $R_{1}$ can be interpreted as the minimum distance between vehicles to ensure safety or the 
range of protected zone as proposed in [6] while the upper limit $R_{2}$ is the maximum transmission distance due to $S$ 's coverage limit.

The received signal at node $X, X \in\{D, E\}$, is expressed as

$$
y_{X}=h_{X} x+n,
$$

where $x$ represents the transmitted signal with energy $E_{s}$, $h_{X}$ represents the channel between node $S$ and node $X$, $n$ denotes the additive white Gaussian noise (AWGN) with power spectral density $N_{0}$, which, without loss of generality, is assumed to be the same for both links. Incorporating the effects of small-scale fading and path loss, the channel gain for transmission distance $d_{X}$ between nodes $S$ and $X$ can be further expressed as follows [14]:

$$
h_{X}=\frac{g_{X}}{\sqrt{1+d_{X}^{\alpha}}}
$$

where $g_{X}$ represents the channel gain following double Rayleigh distribution and $\alpha$ denotes the path loss exponent.

\section{Secrecy Capacity Performance Analysis}

\section{A. Distributions of Instantaneous SNRs}

From (2), the instantaneous signal-to-noise ratio (SNR) received at node $X, X \in\{D, E\}$, can be expressed as

$$
\gamma_{X}=\frac{\left|h_{X}\right|^{2} E_{s}}{N_{0}}=\frac{\left|g_{X}\right|^{2} E_{s}}{\left(1+d_{X}^{\alpha}\right) N_{0}}
$$

With $g_{X}$ being double Rayleigh distributed, the probability density function (PDF) $f_{\left|g_{X}\right|^{2}}(x)$ and cumulative distribution function (CDF) $F_{\left|g_{X}\right|^{2}}(x)$ of the random variable (RV) $\left|g_{X}\right|^{2}$ are given by [15]

$$
f_{\left|g_{X}\right|^{2}}(x)=2 K_{0}(2 \sqrt{x}), F_{\left|g_{X}\right|^{2}}(x)=1-2 \sqrt{x} K_{1}(2 \sqrt{x}) .
$$

Since the distance $d_{D}$ is a constant known to node $S$, the CDF $F_{\gamma_{D}}(\gamma)$ of the instantaneous SNR $\gamma_{D}$ can be readily derived by applying the change of RVs and written as [15]

$$
F_{\gamma_{D}}(\gamma)=1-2 \sqrt{\frac{\left(1+d_{D}^{\alpha}\right) N_{0} \gamma}{E_{s}}} K_{1}\left(2 \sqrt{\frac{\left(1+d_{D}^{\alpha}\right) N_{0} \gamma}{E_{s}}}\right) .
$$

For instantaneous SNR $\gamma_{E}$, besides the double Rayleigh fading, the random location of the node $E$ should also be taken into account while deriving the distribution functions.

Proposition 1: The CDF $F_{\gamma_{E}}(\gamma)$ of the instantaneous SNR $\gamma_{E}$ received by node $E$ given that its distance to node $S$ is any distance between $R_{1}$ and $R_{2}$ with equal probability, can be closely approximated numerically by the expression in (6) at the top of next page, where $w_{\iota}$ and $t_{\iota}$ are the weights and abscissas detailed in [16, Eq. 25.4.30].

Proof: Please refer to Appendix A.

\section{B. Average Secrecy Capacity}

The instantaneous secrecy capacity of the considered system is defined as $C_{s}=\max \left\{\ln \left(1+\gamma_{D}\right)-\ln \left(1+\gamma_{E}\right), 0\right\}$ [2]. The average secrecy capacity $\bar{C}_{s}$ can be obtained from [17]

$$
\bar{C}_{s}=\int_{0}^{\infty} \frac{F_{\gamma_{E}}(\gamma)}{1+\gamma}\left[1-F_{\gamma_{D}}(\gamma)\right] d \gamma
$$

Substituting (5) and (6) into (7) and utilizing the following transformations: $\frac{1}{1+x}=G_{1,1}^{1,1}\left(\left.x\right|_{0} ^{0}\right), K_{v}(x)=$ $\frac{1}{2} G_{0,2}^{2,0}\left(\frac{x^{2}}{4} \mid \frac{v}{2},-\frac{v}{2}\right)$ [13, Eq. 9.3], and the integral of product of Meijer G-functions [18, Eq. (07.34.21.0013.01)], we can obtain the exact expression for ASC in (8) on next page.

Remark 1: Observing (8), the ASC under the eavesdropper location uncertainty is bounded by the first term, which is only related to the legitimate node $D$. Indeed, the ASC is always less than the capacity of the legitimate channel capacity. The second term of (8) demonstrates the impact of the location uncertainty of node $E$ on the ASC, where the parameter $\frac{R_{2}-R_{1}}{2}$ represents the mean uncertainty range and $\frac{R_{2}+R_{1}}{2}$ denotes the mean distance to the source node $S$. From (8), the effects of eavesdropper's location uncertainty on the ASC can be readily evaluated.

The above derived expressions provide the average secrecy capacity and distribution of SNR $\gamma_{E}$ in general cases. Next, we consider the following special cases to provide more insights.

Lemma 1: When the node $S$ gains information on the exact position of node $E$, i.e., mathematically $R_{1}=R_{2} \rightarrow d_{E}$ in (8), the $\operatorname{ASC} \bar{C}_{s}$ can be expressed as

$$
\begin{aligned}
& \bar{C}_{s}=\frac{a}{2} \cdot G_{1,3}^{3,1}\left(\frac{a^{2}}{4} \mid \begin{array}{c}
-\frac{1}{2} \\
\frac{1}{2},-\frac{1}{2},-\frac{1}{2}
\end{array}\right)-\frac{a b}{4} \\
& \cdot G_{1,1: 0,2: 0,2}^{1,1: 2,0: 2,0}\left(\begin{array}{c|c|c|c}
-1 & - & - & a^{2} \\
-1 & \frac{1}{2},-\frac{1}{2} & \frac{1}{2},-\frac{1}{2} & \frac{b^{2}}{4}
\end{array}\right),
\end{aligned}
$$

where $a=2 \sqrt{\frac{\left(1+d_{D}^{\alpha}\right) N_{0}}{E_{s}}}$ and $b=2 \sqrt{\frac{\left(1+d_{E}^{\alpha}\right) N_{0}}{E_{s}}}$.

Proof: When $d_{E}$ is known to node $S$, the $\operatorname{CDF} F_{\gamma_{E}}(\gamma)$ of the SNR $\gamma_{E}$ will have the same form as that in (5). Substituting the CDFs of $\gamma_{D}$ and $\gamma_{E}$ into (7) and utilizing the equalities [18, Eq. (07.34.21.0013.01)] and the solution to the integral of product of three Meijer-G functions in terms of extended generalized bivariate Meijer-G function (EGBMGF) [18, Eq. (07.34.21.0081.01)], we can obtain the result in (9).

Lemma 2: When the node $S$ has information on the position of node $E$ and the transmit SNR $\frac{E_{s}}{N_{0}} \rightarrow \infty$, the asymptotic ASC $\bar{C}_{s}$ can be calculated as

$$
\begin{aligned}
\bar{C}_{s}= & \ln (\mu)\left[1-\sqrt{\rho_{1}} G_{2,2}^{2,2}\left(\rho_{1} \mid \mathbf{a}_{1}\right)\right]+G_{3,3: 0,2: 0,2}^{2,2: 2,0: 2,0}\left(\mathbf{a}_{2}|\mathbf{b}| \mathbf{c} \mid 1, \rho_{1}\right) \\
& \cdot \pi \sqrt{\rho_{1}}+\pi \sqrt{\rho_{1}} G_{3,3: 2: 0,2: 0,2}^{2,2,0}\left(\mathbf{a}_{2}-1|\mathbf{b}| \mathbf{c} \mid 1, \rho_{1}\right)-\ln (\nu) \sqrt{\rho_{2}} \\
& \cdot G_{2,2}^{2,2}\left(\rho_{2} \mid \mathbf{a}_{1}\right)+\pi \sqrt{\rho_{2}} G_{3,3: 0,2: 0,2}^{2,2: 2,0: 0}\left(\mathbf{a}_{2}|\mathbf{b}| \mathbf{c} \mid 1, \rho_{2}\right) \\
& +\pi \sqrt{\rho_{2}} G_{3,3: 0,2: 0,2}^{2,2: 2,0: 2}\left(\mathbf{a}_{2}-1|\mathbf{b}| \mathbf{c} \mid 1, \rho_{2}\right)-2 C
\end{aligned}
$$

where $\mathbf{a}_{1}=\left(\begin{array}{c}-0.5,-0.5 \\ 0.5,-0.5\end{array}\right), \mathbf{a}_{2}=\left(\begin{array}{c}-0.5,-0.5,-1 \\ -0.5,-0.5,-1\end{array}\right), \mathbf{b}=\left(\begin{array}{c}- \\ 0,0\end{array}\right)$, $\mathbf{c}=\left(\begin{array}{c}- \\ 0.5,-0.5\end{array}\right), \mu=\frac{1}{\left(1+d_{D}^{\alpha}\right)}, \nu=\frac{1}{\left(1+d_{E}^{\alpha}\right)}, \rho_{1}=\frac{\mu}{\nu}, \rho_{2}=\frac{\nu}{\mu}$.

Proof: Please refer to Appendix B.

Remark 2: Rewriting (15) leads to the following expression:

$$
\begin{aligned}
\bar{C}_{s}= & \ln \left(\rho_{1}\right) \cdot \int_{0}^{\infty} \int_{\frac{y}{\rho_{1}}}^{\infty} 2 K_{0}(2 \sqrt{y}) \cdot 2 K_{0}(2 \sqrt{x}) d x d y \\
& +\int_{0}^{\infty} \int_{\frac{y}{\rho_{1}}}^{\infty} 2 K_{0}(2 \sqrt{y}) \cdot 2 K_{0}(2 \sqrt{x}) \ln \left(\frac{x}{y}\right) d x d y .
\end{aligned}
$$

Noticing that both integrals in (11) are consistent, we can conclude that the asymptotic ASC follows the scaling law of 


$$
\begin{aligned}
F_{\gamma_{E}}(\gamma) \cong & 1-\frac{\sqrt{\gamma}}{R_{2}+R_{1}}\left(\frac{N_{0}}{E_{s}}\right)^{\frac{1}{2}} \sum_{\iota=0}^{L} w_{\iota} \cdot\left(\frac{R_{2}-R_{1}}{2} t_{\iota}+\frac{R_{2}+R_{1}}{2}\right) \cdot\left[1+\left(\frac{R_{2}-R_{1}}{2} t_{\iota}+\frac{R_{2}+R_{1}}{2}\right)^{\alpha}\right]^{\frac{1}{2}} \\
& \cdot G_{0,2}^{2,0}\left(\frac{N_{0} \gamma}{E_{s}}\left[1+\left(\frac{R_{2}-R_{1}}{2} t_{\iota}+\frac{R_{2}+R_{1}}{2}\right)^{\alpha}\right] \mid \frac{1}{2},-\frac{1}{2}\right) .
\end{aligned}
$$

$$
\begin{aligned}
& \bar{C}_{s} \cong \sqrt{\frac{\left(1+d_{D}^{\alpha}\right) N_{0}}{E_{s}}} G_{1,3}^{3,1}\left(\left.\frac{\left(1+d_{D}^{\alpha}\right) N_{0}}{E_{s}}\right|_{\frac{1}{2},-\frac{1}{2},-\frac{1}{2}} ^{-\frac{1}{2}}\right)-\frac{\left(\frac{N_{0}}{E_{s}}\right)}{R_{2}+R_{1}} \sum_{\iota=0}^{L} w_{\iota}\left(\frac{R_{2}-R_{1}}{2} t_{\iota}+\frac{R_{2}+R_{1}}{2}\right)\left[1+\left(\frac{R_{2}-R_{1}}{2} t_{\iota}+\frac{R_{2}+R_{1}}{2}\right)^{\alpha}\right]^{\frac{1}{2}}
\end{aligned}
$$

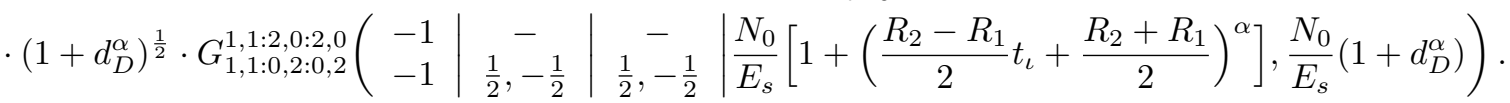

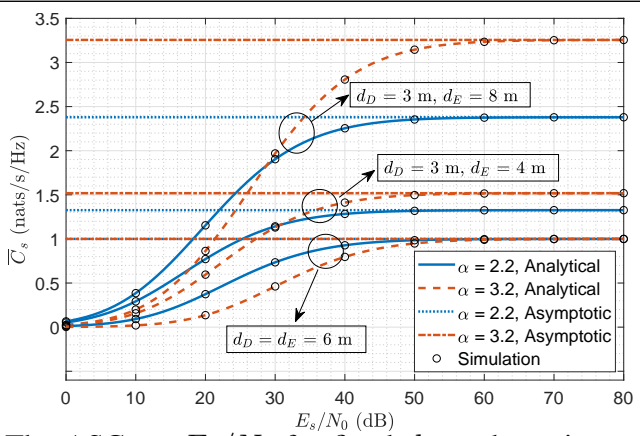

Fig. 1: The ASC vs. $E_{s} / N_{0}$ for fixed $d_{E}$ and varying $\alpha$ values.

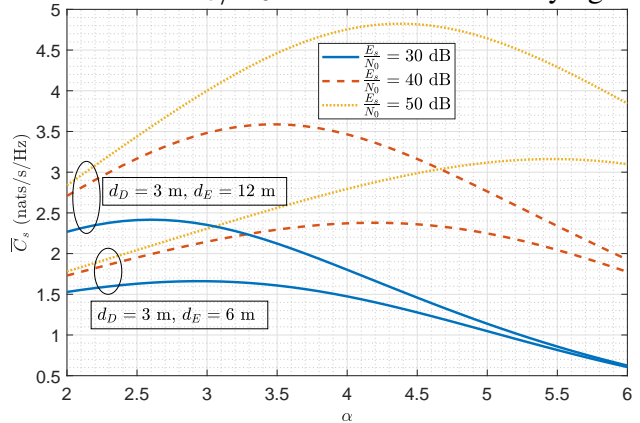

Fig. 3: The ASC vs. path loss exponent $\alpha$ with fixed $E_{s} / N_{0}$ values. F $\Theta\left(\ln \left(\rho_{1}\right)\right)$ (namely $\Theta\left(\ln \left(\frac{1+d_{D}^{\alpha}}{1+d_{E}^{\alpha}}\right)\right)$ ) as $\rho_{1}$ increases and thus depends only on the relative position of the nodes $D$ and $E$.

Remark 3: When the distances $d_{D}$ and $d_{E}$ are equal, namely $\rho_{1}=\rho_{2}=1$ in (10), the asymptotic ASC will always be a constant equaling $1 \mathrm{nat} / \mathrm{s} / \mathrm{Hz}$, which is different from other fading distributions [3, Figs. 1, 2]. This also reaffirms our statement on scaling law of $\Theta\left(\ln \left(\frac{1+d_{D}^{\alpha}}{1+d_{E}^{\alpha}}\right)\right)$ since $\ln (1)=0$.

\section{NumericAl Results AND Discussion}

For the purpose of numerical evaluation, we adopt the following simulation parameters: unless stated otherwise, node $D$ is $d_{D}=3 \mathrm{~m}$ away from node $S$, node $E$ is located somewhere between $R_{1}=4 \mathrm{~m}$ and $R_{2}=12 \mathrm{~m}$ away from $S$.

Figure 1 shows the ASC as a function of the transmit SNR $E_{s} / N_{0}$ for different values of path loss exponent assuming that the location of $E$ is known to $S$. It can be observed that the ASC performance can be improved by increasing the transmit SNR within the low transmit SNR region, whereas the transmit SNR has a limited impact on the ASC in the high transmit SNR region, which is clearly independent of the transmit SNR and is theoretically verified in Lemma 2 . This reveals that the secrecy capacity limit does not depend on the transmit SNR but the relative locations of the nodes, which is confirmed in
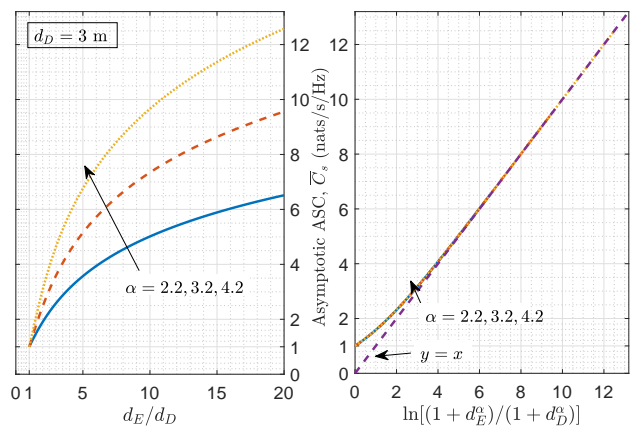

Fig. 2: The asymptotic ASC vs. $d_{E} / d_{D}$ and $\ln \left(\left(1+d_{E}^{\alpha}\right) /\left(1+d_{D}^{\alpha}\right)\right)$.

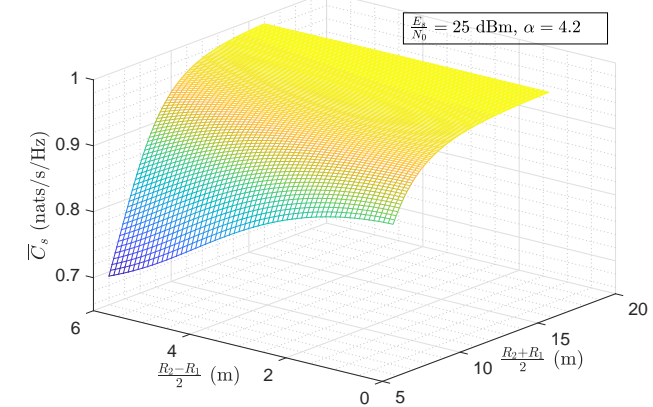

ig. 4: ASC vs. uncertainty range $\frac{R_{2}^{0}-R_{1}}{2}$ and mean uncertainty distance $\frac{R_{2}+R_{1}}{2}$.

Fig. 2. Figure 2 illustrates the ASC versus the ratio $\frac{d_{E}}{d_{D}}$ in the first subplot as well as the scaling law of the asymptotic ASC with respect to $\ln \left(\frac{1+d_{D}^{\alpha}}{1+d_{E}^{\alpha}}\right)$ in the second subplot.

Figure 3 depicts the ASC versus the path loss exponent with fixed values of input SNR. It is clear that a monotonic increasing or decreasing relationship does not necessarily hold between the ASC and the path loss exponent for fixed $E_{s} / N_{0}$ values. This is due to the fact that secrecy capacity depends on the capacity difference of the legitimate and eavesdropper channels even though greater values of path loss exponent indicate less capacity for both channels.

Figure 4 illustrates the impact of the eavesdropper location uncertainty on the ASC performance. It is concluded that when the uncertainty on $E$ 's location decreases (i.e., smaller values of $\frac{R_{2}-R_{1}}{2}$ ), the uncertainty of node $E$ 's received SNR decreases; therefore, the ASC increases, which is also in accordance with the results in [6]. Also, as the node $E$ is statistically located further (i.e., greater values of $\frac{R_{2}+R_{1}}{2}$ ), the ASC increases. Additionally, it is observed that under the condition of eavesdropper location uncertainty, the uncertainty range $\frac{R_{2}-R_{1}}{2}$ poses a greater impact on the ASC when the mean uncertainty distance $\frac{R_{2}+R_{1}}{2}$ is small. The results imply the importance of taking into account the uncertainty of 
eavesdropper's location while designing V2V communication systems with PLS.

\section{Conclusion}

In this letter, the physical layer secrecy capacity over double Rayleigh fading channels were derived and analyzed. The obtained results can be used for the secrecy capacity analysis for $\mathrm{V} 2 \mathrm{~V}$ and $\mathrm{M} 2 \mathrm{M}$ communications as well as for keyhole channels.

\section{Appendix A: Proof of Proposition 1}

We first derive the CDF of RV $\left|h_{E}\right|^{2}$ with location uncertainty of $E$ for $S$. The $\mathrm{CDF} F_{\left|h_{E}\right|^{2}}(x)$ of the $\mathrm{RV}\left|h_{E}\right|^{2}$ is related to the $\mathrm{CDF} F_{\left|g_{E}\right|^{2}}(\cdot)$ of $\mathrm{RV}\left|g_{E}\right|^{2}$ shown in (4) as follows [5]:

$$
\begin{aligned}
F_{\left|h_{E}\right|^{2}}(x)= & \frac{2}{R_{2}^{2}-R_{1}^{2}} \int_{R_{1}}^{R_{2}} z F_{\left|g_{E}\right|^{2}}\left(x\left(1+z^{\alpha}\right)\right) d z=1-\frac{4}{R_{2}^{2}-R_{1}^{2}} \\
& \cdot \int_{R_{1}}^{R_{2}} z \sqrt{x\left(1+z^{\alpha}\right)} K_{1}\left(2 \sqrt{x\left(1+z^{\alpha}\right)}\right) d z .
\end{aligned}
$$

Expressing the modified Bessel function using Meijer Gfunction [13, Eq. 9.34] and applying the Gauss-Legendre quadrature technique [16, Eq. 25.4.30], the CDF $F_{\left|g_{E}\right|^{2}}(\cdot)$ in (12) can be approximated by

$$
\begin{aligned}
& F_{\left|h_{E}\right|^{2}}(x)=1-\frac{2 \sqrt{x}}{R_{2}^{2}-R_{1}^{2}} \int_{R_{1}}^{R_{2}} \underbrace{z \sqrt{1+z^{\alpha}} G_{0,2}^{2,0}\left(x\left(1+z^{\alpha}\right) \mid \frac{1}{2},-\frac{1}{2}\right)}_{g(z)} d z \\
& \cong 1-\frac{\sqrt{x}}{R_{2}+R_{1}} \sum_{\iota=0}^{L} w_{\iota} \cdot g\left(\frac{R_{2}-R_{1}}{2} t_{\iota}+\frac{R_{2}+R_{1}}{2}\right),
\end{aligned}
$$

where $w_{\iota}$ and $t_{\iota},(\iota=1, \ldots, L)$, are the weights and zeros of the $L$-order Legendre polynomial [16, Eq. 25.4.30]. An arbitrarily accurate approximation can be achieved by choosing the appropriate value of $L$. It should be noted that the modified Bessel function in (12) can also be expressed as $K_{1}(z)=2 \sqrt{\pi} e^{-z} z U(1.5,3,2 z)$ with $U(\cdot, \cdot, \cdot)$ being the Kummer hypergeometric function [13, Eq. 9.2]. The existence of the exponential term $e^{-2 \sqrt{x\left(1+z^{\alpha}\right)}}$ in $K_{1}\left(2 \sqrt{x\left(1+z^{\alpha}\right)}\right)$ implies that (13) can converge rapidly as $L$ increases and only limited terms are required to get satisfactory accuracy.

Finally, utilizing the first equality of (3) in (13), we obtain the CDF $F_{\gamma_{E}}(\gamma)$ of the SNR $\gamma_{E}$ as in Proposition 1.

\section{Appendix B: Proof of Lemma 2}

The ASC can be alternatively expressed as [3]

$$
\bar{C}_{s}=\int_{0}^{\infty} f_{\gamma_{E}}\left(\gamma_{E}\right) \int_{\gamma_{E}}^{\infty} \ln \left(\frac{1+\gamma_{D}}{1+\gamma_{E}}\right) f_{\gamma_{D}}\left(\gamma_{D}\right) d \gamma_{D} d \gamma_{E} \text {. }
$$

For notational simplicity, we make the following changes of RVs: $\gamma_{t}=E_{s} / N_{0}, \mu=1 /\left(1+d_{D}^{\alpha}\right), \nu=1 /\left(1+d_{E}^{\alpha}\right), \rho_{1}=\frac{\mu}{\nu}$, $\rho_{2}=\frac{\nu}{\mu}, x=\left|h_{D}\right|^{2}$, and $y=\left|h_{E}\right|^{2}$; and the SNRs can be written as: $\gamma_{D}=\gamma_{t} \mu x$ and $\gamma_{E}=\gamma_{t} \nu y$. Then, the ASC can be expressed after some mathematical manipulations as

$$
\begin{gathered}
\bar{C}_{s}=4 \int_{0}^{\infty} K_{0}(2 \sqrt{y}) \int_{\rho_{2} y}^{\infty} \ln \left(1+\frac{\mu x-\nu y}{\frac{1}{\gamma_{t}}+\nu y}\right) K_{0}(2 \sqrt{x}) d x d y \\
\stackrel{\gamma_{\mathrm{t}} \rightarrow \infty}{\approx} 4 \int_{0}^{\infty} \int_{\rho_{2} y}^{\infty} K_{0}(2 \sqrt{y}) \ln (\mu x) K_{0}(2 \sqrt{x}) d x d y-4 \int_{0}^{\infty} \int_{\rho_{2} y}^{\infty} \\
K_{0}(2 \sqrt{y}) \ln (\nu y) K_{0}(2 \sqrt{x}) d x d y .
\end{gathered}
$$

Denoting the first integral in (15) by $I_{1}$ and second one by $I_{2}$; after changing order of integration for $I_{1}$ and applying the equality [13, Eq. 6.561.4], we can have

$$
\begin{aligned}
& I_{1}=4 \int_{0}^{\infty} \ln (\mu x) K_{0}(2 \sqrt{x})\left[\frac{1}{2}-\sqrt{\rho_{1} x} K_{1}\left(2 \sqrt{\rho_{1} x}\right)\right] d x, \\
& I_{2}=4 \int_{0}^{\infty} \ln (\nu y) K_{0}(2 \sqrt{y}) \sqrt{\rho_{2} y} K_{1}\left(2 \sqrt{\rho_{2} y}\right) d y .
\end{aligned}
$$

Finally, representing the functions in the (16) and (17) through Meijer-G functions and applying integrals of product of Meijer G-functions in [18, Eqs. (07.34.21.0081.01), (07.34.21.0013.01)], we obtain the asymptotic ASC in (10).

In (10), The Euler constant $C$ comes from the integral in (16): (a) $\int_{0}^{\infty} \ln (x) K_{0}(2 \sqrt{x})=-C$. To prove the relation, we first consider the Mellin transform of $K_{0}(2 \sqrt{t})$ : (b) $\int_{0}^{\infty} t^{s-1} K_{0}(t) d x=2^{s-1} \Gamma\left(\frac{s}{2}\right)^{2}$ [13, Eq. 12.43.18]. Differentiating the equality (b) and setting $s=2$ leads to (c) $\int_{0}^{\infty} t \ln (t) K_{0}(t) d t=-C+\ln (2)$. Finally, substituting $t=$ $2 \sqrt{x}$ in (c) and applying the equality: $\int_{0}^{\infty} K_{0}(2 \sqrt{x}) d x=0.5$, we can obtain the equality after some straightforward algebra.

\section{REFERENCES}

[1] A. Hyadi et al., "An overview of physical layer security in wireless communication systems with CSIT uncertainty," IEEE Access, vol. 4, pp. 6121-6132, Sept. 2016.

[2] N. Bhargav et al., "Secrecy capacity analysis over $\kappa-\mu$ fading channels: Theory and applications," IEEE Trans. Commun., vol. 64, no. 7, pp 3011-3024, Jul. 2016.

[3] H. Lei et al., "Secrecy capacity analysis over $\alpha-\mu$ fading channels," IEEE Commun. Lett., vol. 21, no. 6, pp. 1445-1448, Jun. 2017.

[4] G. C. Alexandropoulos and K. P. Peppas, "Secrecy outage analysis over correlated composite Nakagami-m/Gamma fading channels," IEEE Commun. Lett., vol. 22, no. 1, pp. 77-80, Jan. 2018.

[5] D. S. Karas et al., "Physical layer security with uncertainty on the location of the eavesdropper," IEEE Wireless Commun. Lett., vol. 5, no. 5 , pp. $540-543$, Oct. 2016.

[6] W. Liu et al., "On ergodic secrecy capacity of random wireless networks with protected zones," IEEE Trans. Veh. Technol., vol. 65, no. 8, pp. 6146-6158, Aug. 2016.

[7] L. Li et al., "A survey of traffic control with vehicular communications," IEEE Trans. Intell. Transp. Syst., vol. 15, no. 1, pp. 425-432, Feb. 2014.

[8] M. Azees et al., "Comprehensive survey on security services in vehicular ad-hoc networks," IET Intell. Transp. Syst., vol. 10, no. 6, Aug. 2016.

[9] A. S. Akki and F. Haber, "A statistical model of mobile-to-mobile land communication channel," IEEE Trans. Veh. Technol., vol. 35, no. 1, pp. 2-7, Feb. 1986.

[10] V. Erceg et al., "Comparisons of a computer-based propagation prediction tool with experimental data collected in urban microcellular environments," IEEE J. Sel. Areas Commun., vol. 15, no. 4, May 1997.

[11] I. Z. Kovacs, "Radio channel characterisation for private mobile radio systems-mobile-to-mobile radio link investigations," Ph.D. dissertation, Aalborg Universitet, 2002.

[12] D. Chizhik et al., "Multiple-input-multiple-output measurements and modeling in Manhattan," IEEE J. Sel. Areas Commun., vol. 21, no. 3, pp. 321-331, Apr. 2003.

[13] I. S. Gradshteyn and I. M. Ryzhik, Table of Integrals, Series, and Products. Academic Press, 2007.

[14] A. Tukmanov et al., "Outage performance analysis of imperfect-CSIbased selection cooperation in random networks," IEEE Trans. Commun., vol. 62, no. 8, pp. 2747-2757, Aug. 2014.

[15] M. Seyfi et al., "Relay selection in dual-hop vehicular networks," IEEE Signal Process. Lett., vol. 18, no. 2, pp. 134-137, Feb. 2011.

[16] M. Abramowitz and I. A. Stegun, Handbook of Mathematical Functions: With Formulas, Graphs, and Mathematical Tables. Courier Corporation, 1964, vol. 55.

[17] H. Lei et al., "On secrecy performance of mixed RF-FSO systems," IEEE Photon. J., vol. 9, no. 4, pp. 1-14, Aug. 2017.

[18] The Wolfram Functions Site. [Online]. Available: http://functions. wolfram.com/ 